\title{
Testes de mobilidade de chumbo e arsênio em solo contaminado em Apiaí (SP)
}

\section{Juliana Martins ${ }^{1}$ \\ Bernardino Ribeiro Figueiredo ${ }^{1 *}$ \\ 'Universidade Estadual de Campinas - \\ Campinas (SP), Brasil.}

*Autor correspondente: berna@ige.unicamp.br

\section{Resumo}

A mobilidade de chumbo ( $\mathrm{Pb}$ ) e arsênio (As) em solo é avaliada neste trabalho por meio de ensaios geoquímicos. A área estudada localiza-se em Apiaí, no estado de São Paulo, onde se situam as ruínas de uma antiga usina de refino de minério de chumbo e prata e depósitos antigos de escória. $\mathrm{Na}$ área predomina o latossolo vermelho com teores de $\mathrm{Pb}$ e de As bem superiores aos valores regulamentados no Brasil. Este estudo contemplou a determinação do $\mathrm{pH}$ do solo e conteúdo de matéria orgânica (MO) bem como a determinação da composição mineralógica e química do solo por Difração de Raios X e Fluorescência de Raios X, respectivamente. Os solos são derivados predominantemente de rochas básicas e apresentam teores de até $5,1 \% \mathrm{~Pb}$ e $1,3 \%$ As. Foram realizados ensaios de solubilidade e de extração parcial com acetato de amônio em 26 amostras de solo e 1 de escória. Os extratos foram analisados por Espectrometria de Massa com fonte de plasma acoplado indutivamente (ICP-MS). As frações solúveis apresentaram concentrações baixas de $\mathrm{Pb}$ e As ( $<0,1 \%$ dos conteúdos totais em solo) enquanto os extratos parciais apresentaram concentrações nos intervalos $6,9-71,6 \% \mathrm{~Pb}$ e $0,3-2,3 \%$ As em relação aos teores totais das amostras de solo. A mobilidade dessas substâncias ocorre em função de parâmetros como pH do solo, conteúdo de $\mathrm{MO}$, presença de óxidos-hidróxidos de ferro e minerais de argila. Estes resultados evidenciam restrições para o uso de água subterrânea e para o plantio de alimento na área, além da necessidade de adoção de medidas de proteção ambiental compatíveis com o uso da propriedade para visitação pública.

Palavras-chave: chumbo, arsênio, geoquímica de solo, Apiaí.

\section{Abstract}

The mobility of lead $(\mathrm{Pb})$ and arsenic (As) in soil was assessed by carrying out geochemical essays. Soil samples were collected in an area located in the Apiai city, São Paulo State, Brazil, where ruins of a disabled smelt plant for lead and silver and old slag deposits are found. The dominant soil type is red oxisol with $\mathrm{Pb}$ and As contents exceeding regulated values in Brazil. This study included determination of soil $p H$ and its organic matter content as well as determination of the mineral and chemical compositions of soil by X-Ray Diffraction and X-Ray Fluorescence, respectively. Soil is mostly derived from basic rocks and contains up to $5.1 \% \mathrm{~Pb}$ e $1.3 \%$ As. The experiments consisted of solubilization in deionized water and of partial digestion with ammonium acetate of 26 soil samples and 1 sample of slag. Resulting solutions were analyzed by ICP-MS. Soluble fractions revealed very low $\mathrm{Pb}$ and As contents $(<0.1 \%$ of total contents in soil) whereas partial extraction fractions yielded concentrations in the intervals $6.9-71.6 \% \mathrm{~Pb}$ and $0.3-2.3 \%$ As 
of total contents in soil. The results collectively indicate that mobility of $\mathrm{Pb}$ and As in soil occurs as a function of soil $p H$, organic matter content and the presence of iron oxides and hydroxides and clay minerals. They also indicate that local people should avoid consumption of groundwater and edible crops. In addition, appropriate environmental protection actions should be adopted in the area to make it safe for public use.

Keywords: lead, arsenic, soil geochemistry, Apiaí.

\section{INTRODUÇÃO}

Os estudos sobre a ocorrência de metais e de outras substâncias tóxicas em solo vêm se desenvolvendo há muito tempo e têm contribuído para a melhor caracterização de áreas contaminadas com vistas à formulação de estratégias de proteção à saúde do ambiente e das pessoas. No presente estudo foram utilizadas ferramentas da geoquímica para analisar a mobilidade de chumbo $(\mathrm{Pb})$ e arsênio (As) em solo tropical severamente contaminado por essas substâncias.

A área de estudo localiza-se no Centro Integrado de Ensino Multidisciplinar (CIEM/Companhia de Pesquisa de Recursos Minerais - Serviço Geológico do Brasil), Apiaí, São Paulo, onde ocorrem visitas e se realizam atividades destinadas aos moradores e público em geral (Chieregati et al. 2009).

Nesse local, segundo Santos (1960), operou a Usina Experimental de Chumbo e Prata, denominada Usina do Calabouço, no período de 1940 a 1956. Como resultado do beneficiamento e fundição de minério de $\mathrm{Pb}$ foi gerado passivo ambiental constituído de poeiras minerais, resíduos metálicos e depósitos de escória. Essa área foi anteriormente estudada por Martins (2009), que encontrou concentrações $\mathrm{de} \mathrm{Pb}$ e As em solo muito superiores aos valores de referência regulamentados (CONAMA 2009).

A exposição humana ao $\mathrm{Pb}$ e As pode conduzir a sérios agravos à saúde, o que explica o fato desses elementos se destacarem no topo da lista de substâncias perigosas Priority List of Hazardous Substances da Agency for Toxic Substances and Disease Registry (ATSDR 2013).

O presente estudo foi orientado para reunir informação sobre os processos que regem a mobilidade desses elementos em solo tropical por meio de testes geoquímicos e também para contribuir para a apreciação do risco para a saúde na área de estudo.

A área de estudo está localizada na cidade de Apiaí, no Vale do Ribeira, estado de São Paulo (24³0'32"S e $48^{\circ} 50^{\prime} 34^{\prime}$ W) a aproximadamente $326 \mathrm{~km}$ da cidade de São Paulo e $120 \mathrm{~km}$ de Curitiba, sendo as principais vias de acesso a SP-250, SP-165 e a BR 476, que liga Curitiba a Adrianópolis (Figura 1).

A região de Apiaí foi palco de atividade mineradora e beneficiamento de minerais nas décadas de 1930 e 1940, com destaque para a Usina Experimental do Calabouço, no Bairro Palmital, dedicada ao refino do minério de $\mathrm{Pb}$ e prata, provenientes das minas Furnas, Lajeado e de Panelas, a última pertencente à Sociedade Mineração Plumbum Ltda, Adrianópolis, estado do Paraná (Luz 1996).
Embora o Vale do Ribeira já tenha sido objeto de inúmeros estudos ambientais, a pesquisa realizada por Martins (2009) representou o primeiro registro de contaminação $\mathrm{de} \mathrm{Pb}$ nessa localidade em Apiaí. Segundo esse trabalho, os valores de concentração total de metais em solo alcançam na área contaminada níveis bem superiores aos toleráveis para solo conforme a Resolução 420 do Conselho Nacional do Meio Ambiente (CONAMA 2009). No minério processado na Usina do Calabouço, o $\mathrm{Pb}$ ocorre predominantemente como o mineral galena (PbS), em associação com outros sulfetos de ferro, cobre e zinco e quantidades menores de arsenopirita (FeAsS), entre outros minerais.

$\mathrm{Na}$ área de estudo predomina o latossolo vermelho, derivado de alteração de rochas básicas (Gabro Apiaí). Mais subordinadamente ocorre latossolo amarelo, derivado de rocha metassedimentar da Formação Gorutuba. Essas rochas que afloram na área de estudo fazem parte do Grupo Açungui da Faixa de Apiaí (Campanha \& Sadowski 1999, CPRM 2009).

A mobilidade de metais em solo é função de vários parâmetros físicos e químicos, como $\mathrm{pH}$, potencial redox, composição mineralógica, quantidade de sítios ativos para adsorção em óxidos-hidróxidos de Fe, Mn e Al, presença de matéria orgânica (MO) e minerais de argila (Licht 1998).

Especificamente, $\mathrm{o} \mathrm{Pb}$ ocorre no solo, formando principalmente compostos na forma bivalente $\left(\mathrm{Pb}^{2+}\right)$ (Kabata-Pendias 2000). Porém, são vários os fatores que interferem na distribuição do $\mathrm{Pb}$ no solo e na disponibilidade do metal, tais como $\mathrm{pH}$, composição mineral e química do solo, quantidade e tipo de MO, presença de coloides inorgânicos e óxidos de ferro e capacidade de troca iônica do solo. $\mathrm{Na}$ maioria dos casos, o $\mathrm{Pb}$ é retido fortemente no solo como fase ligada à $\mathrm{MO}$, minerais de argila e óxidos-hidróxidos de ferro. $\mathrm{O} \mathrm{Pb}$ pode ser transportado para a água durante o processo de erosão e lixiviação do solo, em locais de alta concentração do metal, excedendo a capacidade de troca cationica e de adsorção do meio. Algumas plantas podem absorver o metal, em especial pelas raízes, nas áreas ricas em $\mathrm{Pb}$.

A exposição crônica ao $\mathrm{Pb}$ provoca efeitos a longo prazo no organismo, chegando a afetar os ossos, rins, o sistema nervoso central, sistemas cardiovascular e reprodutivo, fígado, sistema endócrino e a formação do feto. O elemento não é comprovadamente carcinogênico, porém situa-se entre os metais mais nocivos ao organismo devido aos efeitos neurotóxicos que podem produzir principalmente em crianças (ATSDR 2007a). 
Figura 1

Mapa de localização de Apiaí, no Vale do Ribeira, Estado de São Paulo.

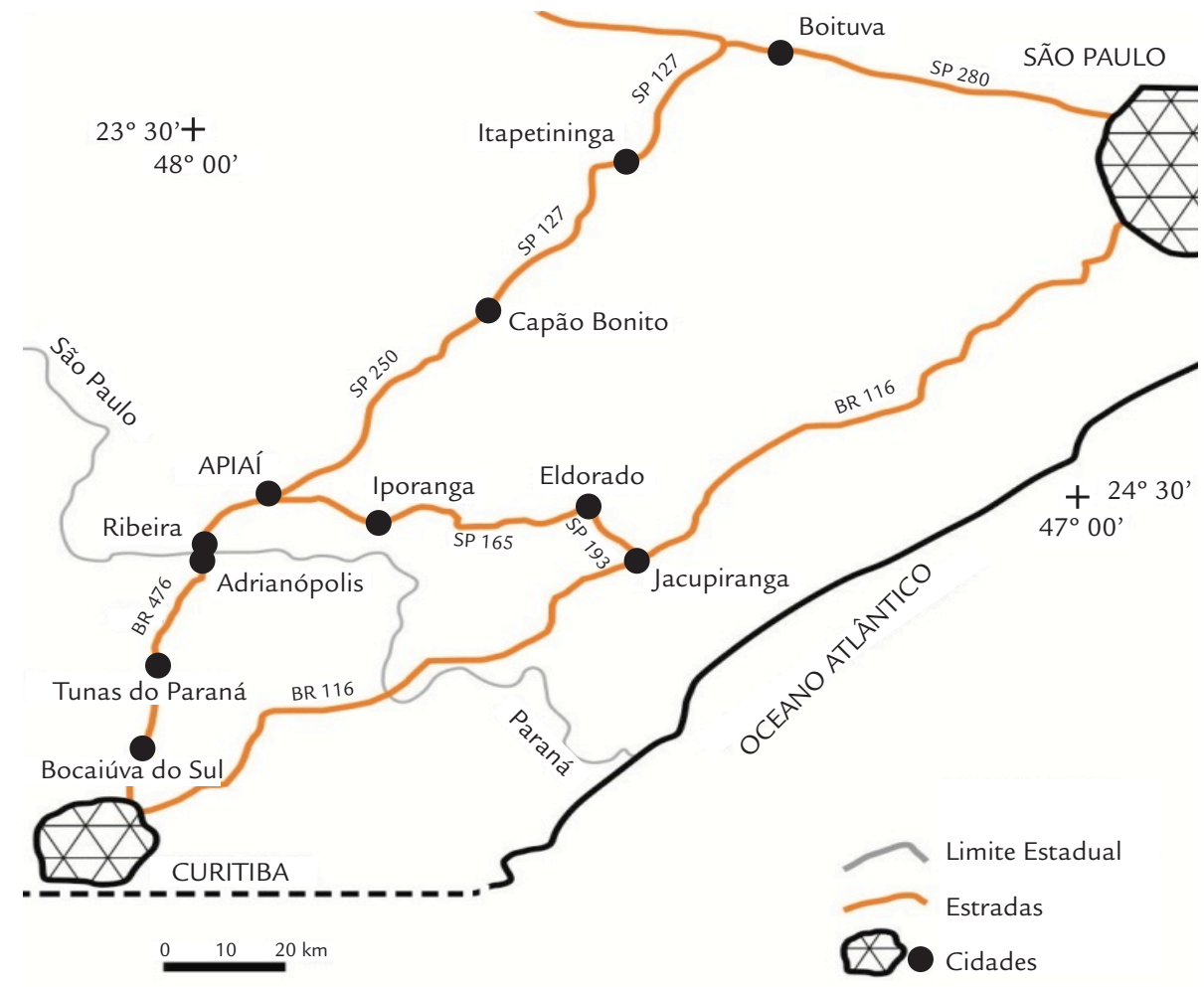

Na população, o grupo que está sujeito ao maior risco de exposição ao $\mathrm{Pb}$ é composto por crianças maiores de seis anos devido às características comportamentais (atividades ao ar livre, contato com solo e hábito de colocar a mão na boca), ao metabolismo ser mais alto que nos adultos, ao fato da absorção de $\mathrm{Pb}$ no trato gastrointestinal ser substancialmente mais alta em crianças que em adultos (em média $50 \%$; nos adultos é $10 \%$ ) e de haver maior deficiência de nutrientes nas crianças, como é o caso de ferro e vitamina $\mathrm{D}$, aumentando, assim, a absorção de $\mathrm{Pb}$ no trato gastrointestinal, entre outros fatores (WHO 1995).

O As ocorre na natureza em diferentes estados de oxidação, como arsenato (5+), arsenito (3+), arsina (3-) e metal (0). Os complexos de $\mathrm{As}(5+)$ e $\mathrm{As}(3+)$ são as espécies mais solúveis e as mais abundantes no solo, sendo os compostos de $\operatorname{As}(3+)$ dezenas de vezes mais tóxicos que os de As $(5+)$ (Sakuma et al. 2002). A presença e as quantidades relativas das diferentes formas de As no solo variam com o pH, potencial redox, composição e textura do solo e presença de microorganismos (Tuperinem et al. 1999).

$\mathrm{O} \mathrm{pH}$ e o potencial redox são importantes na avaliação do destino de As no solo. Em condições oxidantes, o arsenato predomina e a mobilidade de As é baixa. À medida que aumenta o $\mathrm{pH}$ ou o potencial redox diminui, o arsenito predomina. A forma reduzida de As está mais sujeita à lixiviação devido à sua maior solubilidade. Nas condições de solo com muita $\mathrm{MO}$, temperatura e umidade adequadas e outras condições favoráveis à atividade microbiana, a sequência de reação ocorre no sentido da metilação e da volatilização. Arsenito pode ser oxidado a arsenato e os óxidos de manganês são apontados como os principais receptores de elétrons nesta reação de oxidação (McLean \& Bledsoe 1992).

A fonte do As pode ser natural, pois o elemento está presente nos minerais formadores das rochas, incluindo os óxidos de ferro e as argilas, mas principalmente em sulfetos, especialmente em FeAsS e pirita. Pode ser liberado para o meio ambiente por emissões vulcânicas e a partir de fontes antrópicas, como as usinas de fundição de metais não ferrosos e mineração (Chilvers \& Peterson 1987, Selinus 2005, Figueiredo et al. 2007).

O comportamento de arsenato no solo é análogo ao do fosfato devido à sua semelhança química, podendo formar precipitados insolúveis com o ferro, o alumínio e o cálcio. A presença de ferro em solo é eficaz no controle da mobilidade do arsenato. A adsorção do arsenato por caulinita e montmorilonita alcança máximo a pH 3 a 4, seguidos por diminuição gradual da adsorção, com aumento do pH (McLean \& Bledsoe 1992). A adsorção de arsenito também é fortemente dependente do $\mathrm{pH}$. A adsorção por caulinita e montmorilonita é mais alta na faixa de $\mathrm{pH}$ de 3 a 9, atingindo máximo de adsorção por óxido de ferro a pH 7 (McLean \& Bledsoe 1992).

A exposição crônica ao As pode provocar manchas na pele (queratose e hiperqueratose) e também câncer de pele em humanos. O risco de desenvolver outros tipos de câncer no fígado, rins e pulmão também aumenta com a exposição ao As (ATSDR 2007b, Smith \& Huyck 1999, Sakuma et al. 2002). Além dos efeitos dermatológicos, outros não carcinogênicos da exposição humana prolongada a pequenas doses de As têm sido apontados por esses autores, entre eles hipertensão arterial e alterações vasculares e renais. 


\section{MATERIAIS E MÉTODOS}

Amostras de solo foram coletadas em uma malha cobrindo toda a propriedade do CIEM/CPRM, em Apiaí, conforme indicado na Figura 2. Ao todo foram coletadas 26 amostras de solo (0 a $40 \mathrm{~cm}$ ) para análise química e ensaios de mobilidade de metais. Uma amostra de escória, coletada na área da antiga usina, também foi analisada.

Do grupo de amostras de solo, cinco foram selecionadas para ensaios adicionais e determinação da composição mineral por difratometria de raios-X (DRX). O equipamento utilizado foi um difratômetro Siemens D5000 (DPM-UNESP/Rio Claro), operando com radiação de Co K $\alpha$, a $35 \mathrm{~V}$ e $25 \mathrm{~mA}$, com filtro de $\mathrm{Cu}$ e velocidade de goniômetro de $3^{\circ}(2 \theta) / \mathrm{min}$. As fases minerais foram identificadas utilizando-se o software Diffrac-at, V3.0 de SOCABIM-SIEMENS, 1991.

Essas cinco amostras também foram usadas para determinação de $\mathrm{pH}$ e conteúdo de $\mathrm{MO}$ em solo. A análise de $\mathrm{pH}$ do solo (fração granulométrica $<2 \mathrm{~mm}$ ) foi realizada conforme os procedimentos da Empresa Brasileira de Pesquisa Agropecuária - EMBRAPA (1997), com medição da concentração efetiva de íons $\mathrm{H}^{+}$na solução do solo utilizando-se eletrodo combinado, imerso em suspensão solo-água na proporção de 1:2.
Os conteúdos de $\mathrm{MO}$ no solo foram determinados pelo método de Walkley e Black, adaptado de EMBRAPA (1997). Segundo ele, o carbono do solo é oxidado por uma solução oxidante $\left(\mathrm{K}_{2} \mathrm{Cr}_{2} \mathrm{O}^{7}{ }^{2-}\right.$ em meio ácido, $\mathrm{H}_{2} \mathrm{SO}_{4}$ concentrado) e a titulação do dicromato de potássio é feita com uma solução de ferro reduzido $\left(\mathrm{FeSO}_{4} \cdot 7 \mathrm{H}_{2} \mathrm{O}\right)$ em meio ácido, empregando-se como indicador a difenilamina. A partir desta titulação obtêm-se os valores em porcentagem para carbono orgânico (CO), usados para o cálculo da porcentagem de MO pela fórmula: $\mathrm{MO}=\mathrm{CO}(\%) \mathrm{x} 1,724$, em que 1,724 é o fator de correção de Van Bermmelen. O controle de qualidade dessas análises foi monitorado por meio da análise do material de referência 2709 (San Joaquin Soil).

Todas as amostras de solo foram analisadas para a composição química total e utilizadas na realização dos ensaios de solubilidade e extração parcial. Essas análises foram conduzidas na fração granulométrica $<180 \mu \mathrm{m}$ após secagem das amostras ao natural. As alíquotas para análise foram colhidas após o quarteamento das amostras e moagem em moinho de ágata.

As análises químicas totais das amostras de solo e de escória foram realizadas para 10 óxidos maiores e 18 ele-

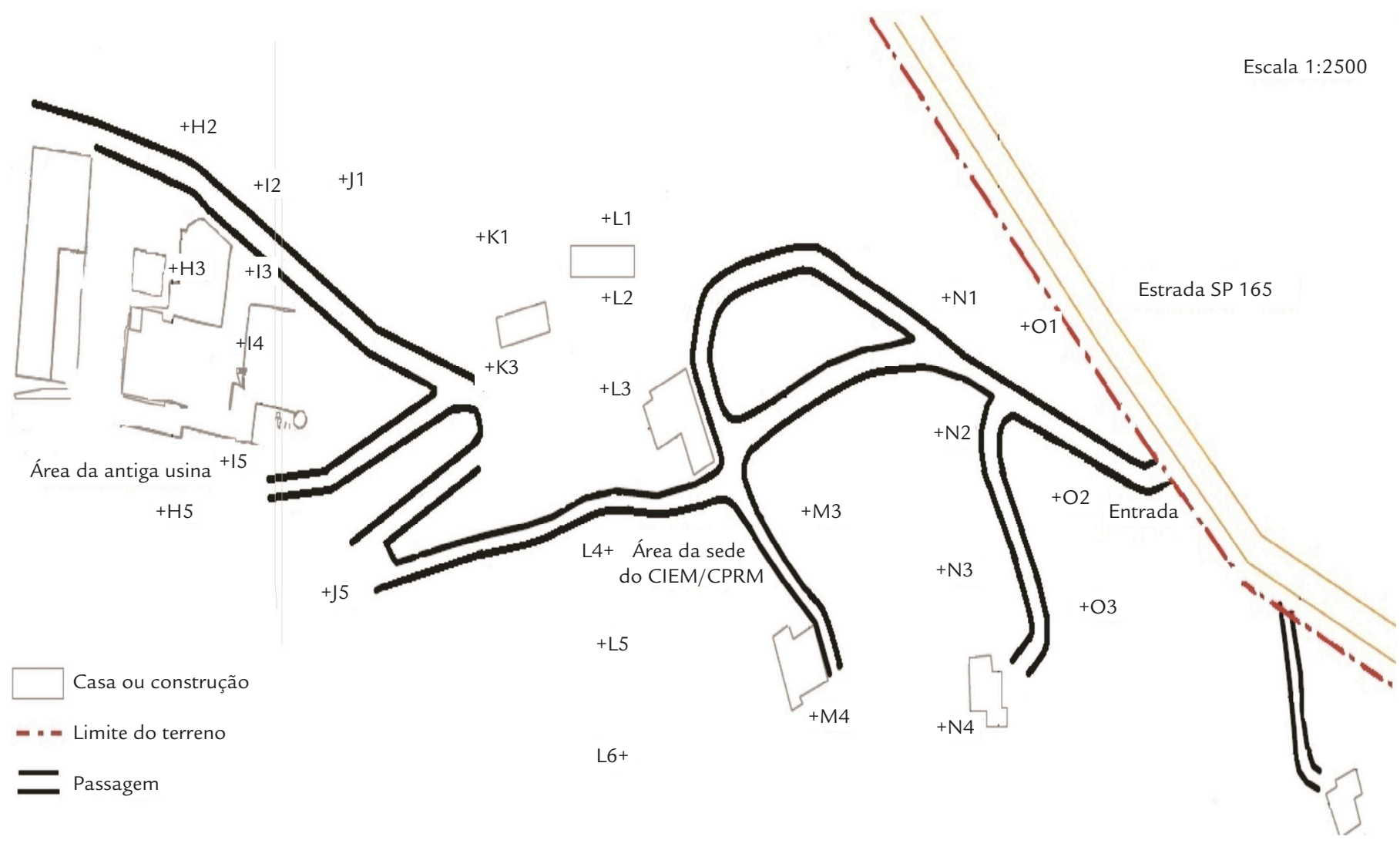

Figura 2

Mapa da área de estudo e localização das amostras de solo. 
mentos menores e elementos-traço em pastilhas prensadas em espectrômetro de fluorescência de raios-X sequencial (PW2404, Philips, IG-UNICAMP), equipado com tubo de ródio. Os percentuais de perda ao fogo dessas amostras foram determinados a $105^{\circ} \mathrm{C}$ e a $1.000^{\circ} \mathrm{C}$. Para o controle de qualidade dos resultados foram analisadas amostras duplicatas e replicatas e, em paralelo, materiais de referência internacionais (GSS-2 e GSS-5). As concentrações de óxidos maiores e menores (exceto $\mathrm{Na}_{2} \mathrm{O}$ e $\mathrm{MgO}$ ) foram calculadas pelo programa Uniquant, e as dos demais elementos pelo programa Solo2010 - Superq.

As amostras de solo e de escória foram submetidas a ensaios de solubilidade e extração parcial, como segue. A extração solúvel foi preparada com base no protocolo proposto em Embrapa (1979) com adaptação de Cunha (2003). Utilizaram-se $10 \mathrm{~g}$ de amostra de solo em um tubo de centrífuga, o qual foi completado até $50 \mathrm{~mL}$ com água Milli-Q. O tubo de ensaio foi agitado cinco vezes ao dia manualmente e vigorosamente por um minuto, ficando uma noite de descanso. No dia seguinte, os tubos foram centrifugados por 10 minutos a $4.000 \mathrm{rpm}$, a solução foi filtrada (filtros com porosidade $0,45 \mu \mathrm{m}$ ) e armazenada em outro tubo de ensaio. $\mathrm{O}$ mesmo procedimento foi utilizado para o branco analítico.

O procedimento do ataque parcial foi adaptado do protocolo de análises de extrações seletivas de Sandström (1984). Utilizaram-se $5 \mathrm{~g}$ de amostra de solo e $30 \mathrm{~mL}$ de acetato de amônio (ajustado para pH 4,5 com ácido acético). Em um tubo de centrífuga, colocou-se a amostra de solo e foram adicionados $20 \mathrm{~mL}$ de $\mathrm{NH}_{4} \mathrm{OAc} 1 \mathrm{M}$. Os tubos foram agitados por 1 minuto manualmente e vigorosamente e então colocados no agitador mecânico planetário por 30 minutos com velocidade $8,5 \mathrm{rpm}$, e depois centrifugados por 10 minutos a $4.000 \mathrm{rpm}$. Após filtragem da solução, adicionaram-se $10 \mathrm{~mL}$ de $\mathrm{NH}_{4} \mathrm{OAc} 1 \mathrm{M}$ ao precipitado no tubo de centrífuga, o qual foi novamente agitado conforme descrito acima, seguido de filtração. Para o branco, usou-se o mesmo procedimento.
Embora em alguns estudos o acetato de amônio seja usado a $\mathrm{pH} 7$, neste trabalho o ácido foi aplicado em solução a pH 4,5. Nessa condição, o ácido mobiliza conjuntamente a fração solúvel e a fração de íons trocáveis, além de uma parte da fração carbonato (Cardoso Fonseca \& Martim 1986, Dold \& Fontboté 2001). Esse método foi empregado por Gatehouse et al. (1977), Sondag (1981), Cardoso Fonseca e Martim (1986), Fonseca e Silva (1998) e Favas et al. (2005), acoplado à técnica analítica de espectrofotometria de absorção atômica (AAS). O acetato é seletivo para os produtos resultantes da alteração de willemita $\left(\mathrm{Zn}_{2} \mathrm{SiO}_{4}\right)$, smithsonita $\left(\mathrm{ZnCO}_{3}\right)$ e piromorfita $\left(\mathrm{Pb}_{5}\left(\mathrm{PO}_{4}\right)_{3} \mathrm{Cl}\right)$, sendo o $\mathrm{Zn}$ e o $\mathrm{Pb}$ completamente extraídos nestes minerais. $\mathrm{O} \mathrm{NH}_{4} \mathrm{OAc}$ também é quase seletivo para a cerussita $\left(\mathrm{PbCO}_{3}\right)$ e a anglesita $\left(\mathrm{PbSO}_{4}\right)$, podendo extrair até $90 \%$ do $\mathrm{Pb}$ nessas fases.

As medições das concentrações elementares nos extratos, tanto na fração solúvel quanto na resultante do ataque parcial, foram realizadas por espectrometria de massa com plasma induzido (Inductively coupled plasma mass spectrometry - ICP-MS), em equipamento XseriesII da Thermo (IG-UNICAMP) equipado com Collision Cell Technology. O controle de qualidade instrumental foi efetuado pela análise do material de referência NIST SRM 1640 (Trace Elements in Natural Water). Foram utilizados ainda os materiais de referência NIST 2710 (Montana Soil - Highly Elevated Trace Element Concentrations) e o NIST 2711 (Montana Soil - Moderately Elevated Trace Element Concentrations).

Neste estudo, o uso de cloreto de magnésio para a extração da fração trocável (Tessier et al. 1979, Bosso \& Enzweiler 2008) foi descartado para evitar a produção de interferências poliatômicas com a massa ${ }^{75} \mathrm{As}$, resultante da formação do composto ${ }^{40} \mathrm{Ar}{ }^{35} \mathrm{Cl}$ durante as análises por ICP-MS (plasma de argônio).

A distribuição espacial dos teores de $\mathrm{Pb}$ e As em solo foram lançadas em mapas de isoteores com a utilização da ferramenta de gridding Inverse Distance to a Power.

\section{RESULTADOS}

\subsection{Caracterização das amostras e determinação de teores de $\mathrm{Pb}$ e As em solo e escória}

A composição mineral do solo foi avaliada por meio da análise de DRX de um grupo de cinco amostras com diferentes teores de $\mathrm{Pb}$ e coletadas em pontos diferentes da área de estudo. Os principais minerais identificados em todas as amostras foram quartzo, illita-montmorillonita, haloisita e goethita. Em quantidades subordinadas foram identificados caulinita, esmectita, alofânio, paligorskita e gibbsita. Entre os metais reconhecidos nos minérios da região, apenas o cobre e zinco exibiram fases minerais próprias superiores ao limite de detecção da técnica, como calcoalumita $(\mathrm{Cu})$ e baumita e kehoeíta $(\mathrm{Zn})$. O Pb e As não apresentaram fases próprias em quantidades superiores ao limite de detecção da técnica analítica, porém, em face dos altos teores desses elementos no solo, admite-se que façam parte de fases amorfas ou ad- sorvidas em outros minerais do solo. Óxidos e hidróxidos de Fe bem como os argilominerais representam fases minerais favoráveis à retenção desses elementos (McLean \& Bledsoe 1992). Na escória foram identificadas a magnetita, plumbojarosita e outras fases menores de sulfato e óxido de $\mathrm{Pb}$, entre elas caracolita e lithargita. Em uma das amostras de solo (I4) com altos teores de $\mathrm{Pb}$ e As também foram detectadas quantidades menores de magnetita e ilmenita.

$\mathrm{O}$ pH do solo e os teores de $\mathrm{MO}$ foram determinados por meio de ensaios analíticos nas cinco amostras de solo. $\mathrm{O}$ pH variou de 4,8 a 6,5, obtendo-se o valor máximo na amostra com os teores mais altos de $\mathrm{Pb}$ e As, os quais provavelmente indicam mistura de solo com material de escória. Os teores de MO foram relativamente constantes nas amos- 
tras de solo e variaram no intervalo de 1,9 a 2,3\% de MO.

Segundo a Agência de Proteção Ambiental dos Estados Unidos da América (USEPA 1996) e a Agency for Toxic Substances and Disease Registry (ATSDR 1999), para valores de $\mathrm{pH}$ entre 6 e 8 e MO inferior a $5 \%$, (exemplo da amostra I4 com alto $\mathrm{Pb}$ e As), há tendência dos solos em formar complexos de óxidos de $\mathrm{Pb}$ hidratados ou, ainda, ocorrer a precipitação na forma de carbonatos ou fosfatos. Já para as outras amostras com pH 4,8 a 5,4 e teores de $\mathrm{MO}$ inferiores a $5 \%$, podem prevalecer condições favoráveis à solubilização dos complexos orgânicos de $\mathrm{Pb}$, favorecendo a absorção do metal pelas plantas, segundo os mesmos autores.

Solos ricos em MO favorecem a ocorrência de concentrações mais altas de As. Em condições aeróbicas, o As ocorre em solos principalmente como arsenato $\left(\mathrm{AsO}_{4}{ }^{3-}\right)$, ligado a minerais de argila, óxidos-hidróxidos de $\mathrm{Fe}$ e $\mathrm{Mn}$ e MO. Segundo Fergusson (1990) e Matschullat (2000), as fases dominantes em solos ácidos são arsenatos de Al e Fe, enquanto o arsenato de cálcio se destaca em solos básicos e calcários.

As concentrações totais de $\mathrm{Pb}$ e As nas amostras de solo e escória constam nas Tabelas 1 e 2, respectivamente. Os teores totais de $\mathrm{Pb}$ variaram de 23 a $51.000 \mathrm{mg} / \mathrm{kg}$, com $61 \%$ das amostras de solo apresentando teores de $\mathrm{Pb}$ superiores ao valor de prevenção $(72 \mathrm{mg} / \mathrm{kg})$ da Resolução 420 do CONAMA (2009). As concentrações totais de As em solo variaram de 3,6 a $6.810 \mathrm{mg} / \mathrm{kg}$, com $42 \%$ das amostras exibindo teores de As superiores ao valor de prevenção $(35 \mathrm{mg} / \mathrm{kg})$ da Resolução 420 do CONAMA (2009). A amostra de escória exibe concentrações de $188.700 \mathrm{mg} / \mathrm{kg} \mathrm{Pb}$ e $15.400 \mathrm{mg} / \mathrm{kg}$ As. Segundo a Resolução 420 do CONAMA (2009), o valor de prevenção (VP) é a concentração de valor limite de determinada substância no solo, sendo capaz de sustentar as suas funções principais. Já o valor de intervenção (VI) é a concentração de determinada substância no solo acima da qual existem riscos potenciais, diretos ou indiretos, à saúde humana, considerando um cenário de exposição padronizado. Os VPs foram utilizados para diferenciar o solo contaminado do não contaminado na área de estudo.

A distribuição espacial das concentrações de $\mathrm{Pb}$ e As em solo são indicadas em mapas de isoteores (Figuras 3 e 4), construídos com base nos dados das Tabelas 1 e 2 .

Como indicado na Figura 3, na área da sede do CIEM/CPRM o solo apresenta teores moderados de $\mathrm{Pb}$, mas, em grande parte, são superiores aos valores de prevenção $(72 \mathrm{mg} / \mathrm{kg})$ e de intervenção para $\mathrm{Pb}$ em solo (180 a $900 \mathrm{mg} / \mathrm{kg}$ ). Na área da usina, as concentrações de $\mathrm{Pb}$ são ainda mais elevadas, podendo alcançar o máximo de $51.000 \mathrm{mg} / \mathrm{kg} \mathrm{Pb}$.

No mapa de isoteores de As em solo (Figura 4) são representados teores de até $500 \mathrm{mg} / \mathrm{kg}$ As na área da sede do CIEM/CPRM, superiores aos valores de intervenção para As em solos que se situam entre 35 e $150 \mathrm{mg} / \mathrm{kg}$ (CONAMA 2009). Já na área da usina, os teores de As em solo são bem altos e alcançam níveis de até $6.810 \mathrm{mg} / \mathrm{kg}$ As.
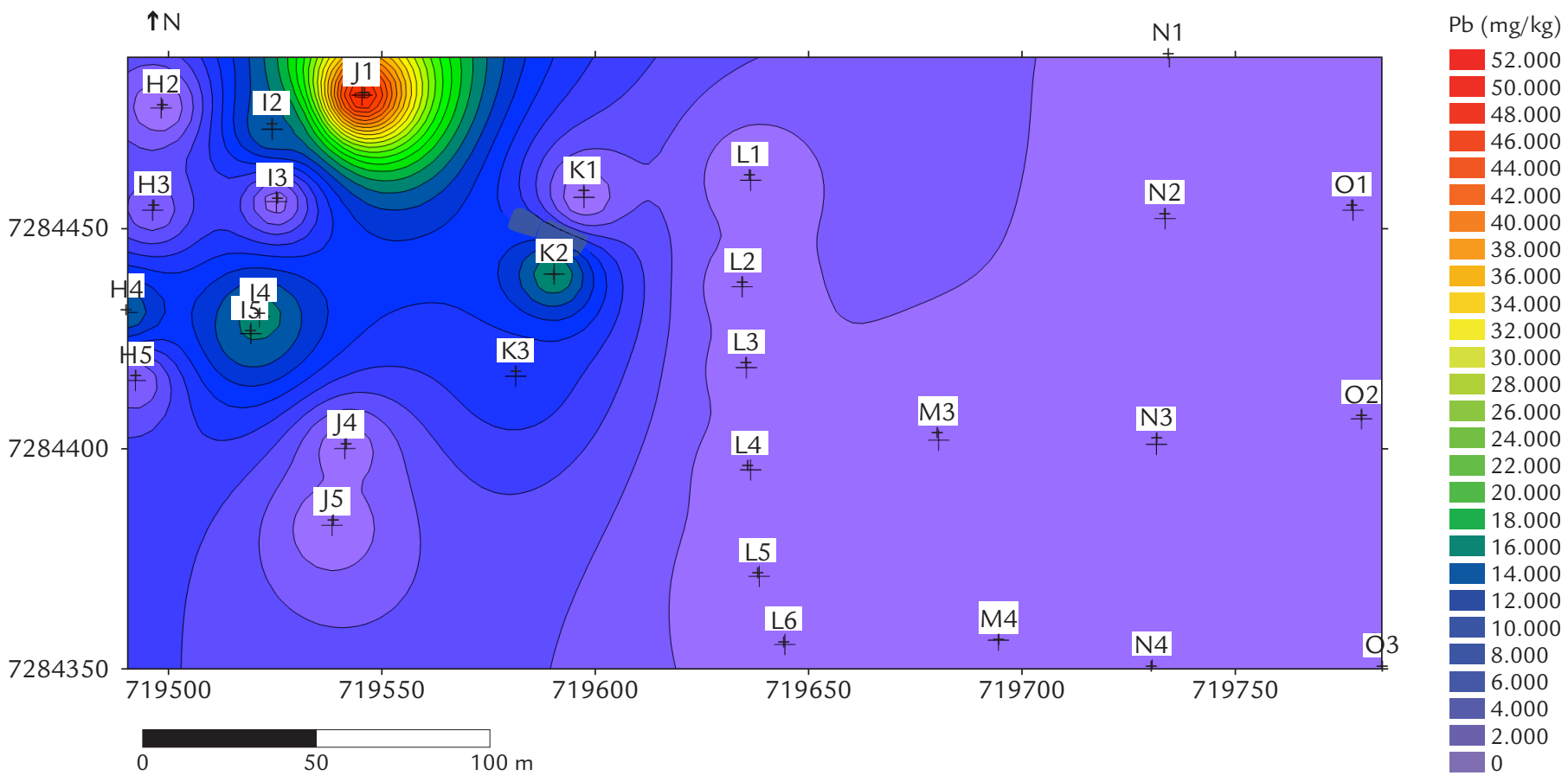

Figura 3

Mapa de isoteores $(\mathrm{mg} / \mathrm{kg})$ de chumbo em solo na área de estudo. 


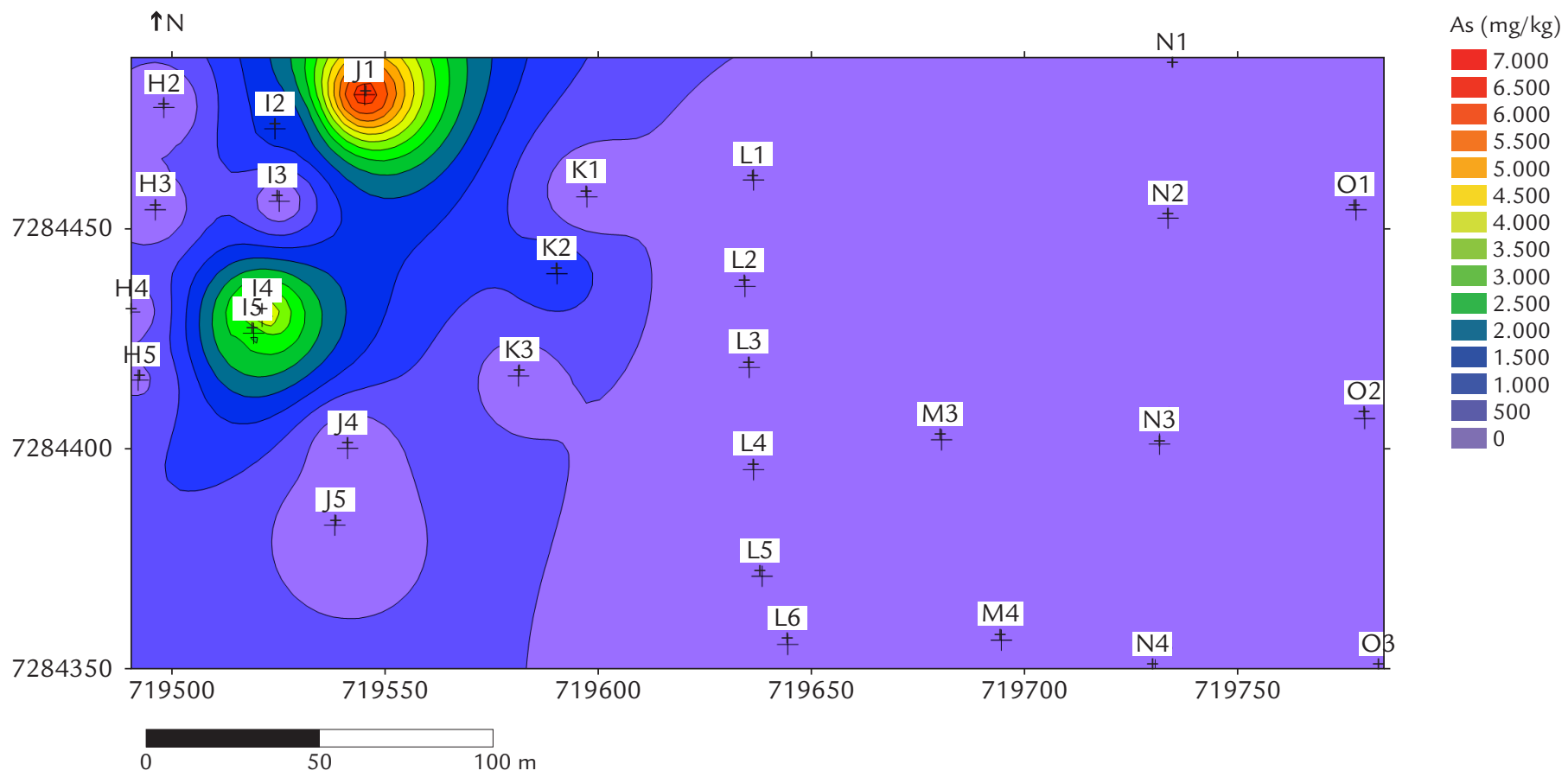

Figura 4

Mapa de isoteores $(\mathrm{mg} / \mathrm{kg})$ de arsênio em solo na área de estudo.

Tabela 1

Concentrações totais de chumbo em amostras de solo e escória de Apiaí (SP) e nas frações solúvel e parcial em massa e em percentagem em relação aos teores totais.

\begin{tabular}{|c|c|c|c|c|c|}
\hline \multirow[t]{2}{*}{ Amostra } & \multirow{2}{*}{$\begin{array}{c}\text { Pb total } \\
\text { FRX } \\
\mathrm{mg} / \mathrm{kg}\end{array}$} & \multicolumn{2}{|c|}{$\begin{array}{l}\text { Pb solúvel } \\
\text { ICP-MS }\end{array}$} & \multicolumn{2}{|c|}{$\begin{array}{l}\text { Pb parcial } \\
\text { ICP-MS }\end{array}$} \\
\hline & & $\mu \mathrm{g} / \mathrm{kg}$ & $\%$ & $\mathrm{mg} / \mathrm{kg}$ & $\%$ \\
\hline O1 & 32 & 15,3 & 0,05 & 10,3 & 32,2 \\
\hline $\mathrm{O} 2$ & 29 & 1,7 & 0,01 & 8,3 & 28,3 \\
\hline O3* & 50 & 25,3 & 0,05 & 7,4 & 14,8 \\
\hline $\mathrm{N} 1{ }^{*}$ & 36 & 0,6 & 0,00 & 2,5 & 6,9 \\
\hline $\mathrm{N} 2$ & 944 & 4,9 & 0,00 & 425 & 45,1 \\
\hline N3 & 52 & 15,1 & 0,00 & 14,7 & 28,3 \\
\hline N4 & 54 & 10,1 & 0,02 & 20 & 37,1 \\
\hline M3 & 23 & 1,8 & 0,01 & 4,8 & 20,7 \\
\hline M4 & 56 & 12,1 & 0,02 & 8,5 & 15,2 \\
\hline L1 & 136 & 0,3 & 0,00 & 21,8 & 16,1 \\
\hline L2 & 593 & 1,7 & 0,00 & 174 & 29,3 \\
\hline L3 & 930 & 4,1 & 0,00 & 362 & 38,9 \\
\hline L4 & 534 & 41,1 & 0,01 & 193 & 36,1 \\
\hline L5 & 31 & 4,2 & 0,01 & 22,2 & 71,6 \\
\hline $\mathrm{L} 6$ & 178 & 27,3 & 0,01 & 49 & 27,5 \\
\hline $\mathrm{K} 1 *$ & 174 & 13,5 & 0,01 & 26,6 & 15,3 \\
\hline $\mathrm{K} 3 * *$ & 9.400 & 98,2 & 0,00 & 4.868 & 51,8 \\
\hline $\mathrm{J} 1 * *$ & 51.000 & 4.499 & 0,01 & 12.240 & 24,1 \\
\hline $\mathrm{J} 5$ & 169 & 45,5 & 0,03 & 52,2 & 30,9 \\
\hline $12 * *$ & 15.700 & 389 & 0,00 & 10.113 & 64,4 \\
\hline $13 * *$ & 782 & 31 & 0,00 & 431 & 55,1 \\
\hline $14^{*, * *}$ & 17.700 & 64,7 & 0,00 & 10.528 & 59,5 \\
\hline $15 * *$ & 16.100 & 254 & 0,00 & 4.755 & 29,5 \\
\hline $\mathrm{H} 2$ & 88 & 17,6 & 0,00 & 38,7 & 43,9 \\
\hline $\mathrm{H} 3 * *$ & 2.405 & 112 & 0,00 & 1.480 & 61,8 \\
\hline $\mathrm{H} 5 * *$ & 1.946 & 125 & 0,01 & 774 & 39,8 \\
\hline Escória** & 188.700 & 0,40 & 0 & 28.058 & 14,9 \\
\hline
\end{tabular}

* Amostras de solo usadas para análises de difratometria de raios- $\mathrm{X}$, $\mathrm{pH}$ e teores de matéria orgânica. ${ }^{*}$ Amostras da área da antiga usina. Pb: chumbo; FRX: fluorescência de raios-X; ICP-MS: Espectrometria de massa com plasma induzido. 


\begin{tabular}{|c|c|c|c|c|c|}
\hline \multirow{2}{*}{ Amostra } & \multirow{2}{*}{$\begin{array}{c}\text { As total } \\
\text { FRX }\end{array}$} & \multicolumn{2}{|c|}{$\begin{array}{l}\text { As solúvel } \\
\text { ICP-MS }\end{array}$} & \multicolumn{2}{|c|}{$\begin{array}{l}\text { As parcial } \\
\text { ICM-MS }\end{array}$} \\
\hline & & $\mu \mathrm{g} / \mathrm{kg}$ & $\%$ & $\mathrm{mg} / \mathrm{kg}$ & $\%$ \\
\hline O1 & 6 & 1,4 & 0,02 & 0,1 & 0,9 \\
\hline $\mathrm{O} 2$ & 4 & 1,6 & 0,04 & 0,1 & 1,1 \\
\hline O3* & 16 & 7,7 & 0,05 & 0,1 & 0,7 \\
\hline $\mathrm{N} 1 *$ & 8 & 1,8 & 0,02 & 0,1 & 0,8 \\
\hline N2 & 91 & 6,9 & 0,01 & 0,3 & 0,3 \\
\hline N3 & 9 & 2,2 & 0,02 & 0,1 & 0,9 \\
\hline $\mathrm{N} 4$ & 9 & 3,7 & 0,04 & 0,1 & 0,6 \\
\hline M3 & 5 & 0,3 & 0,01 & 0,1 & 2,3 \\
\hline M4 & 18 & 6,1 & 0,03 & 0,1 & 0,3 \\
\hline $\mathrm{L} 1$ & 14 & 6,7 & 0,05 & 0,1 & 0,5 \\
\hline L2 & 70 & 9,5 & 0,01 & 0,1 & 0,1 \\
\hline L3 & 42 & 2,6 & 0,01 & 0,2 & 0,6 \\
\hline L4 & 44 & 15,2 & 0,03 & 0,2 & 0,4 \\
\hline L5 & 9,9 & 0,9 & 0,01 & 0,1 & 0,9 \\
\hline L6 & 32 & 11,3 & 0,03 & 0,1 & 0,3 \\
\hline $\mathrm{K} 1 *$ & 53 & 37,2 & 0,07 & 0,2 & 0,3 \\
\hline $\mathrm{K} 3 * *$ & 236 & 8,6 & 0,00 & 4,2 & 1,8 \\
\hline $\mathrm{J} 1 * *$ & 6.810 & 52,6 & 0,01 & 11,1 & 0,2 \\
\hline $\mathrm{J} 5$ & 24 & 4,1 & 0,02 & 0,2 & 0,7 \\
\hline $12 * *$ & 1.550 & 82,4 & 0,01 & 23,1 & 1,5 \\
\hline $13 * *$ & 67 & 15,6 & 0,02 & 0,4 & 0,7 \\
\hline $14^{*}, * *$ & 4.780 & 12,9 & 0,00 & 16,9 & 0,4 \\
\hline $15 * *$ & 2.900 & 91,4 & 0,00 & 5,8 & 0,2 \\
\hline $\mathrm{H} 2$ & 18 & 1,9 & 0,01 & 0,1 & 0,3 \\
\hline $\mathrm{H} 3 * *$ & 71 & 11,5 & 0,02 & 0,5 & 0,8 \\
\hline $\mathrm{H} 5 * *$ & 418 & 36,8 & 0,01 & 0,6 & 0,1 \\
\hline Escória** & 15.400 & 1,33 & 0,01 & 456 & 2,92 \\
\hline
\end{tabular}

*Amostras de solo usadas para análises de difratometria de raios- $\mathrm{X}$, $\mathrm{pH}$ e teores de matéria orgânica. ${ }^{*}$ Amostras da área da antiga usina. As: arsênio; FRX: fluorescência de raios-X; ICP-MS: Espectrometria de massa com plasma induzido.

Tabela 2

Concentrações totais de arsênio em amostras de solo e escória de Apiaí (SP) e nas frações solúvel e parcial em massa e percentagem em relação aos teores totais.

\subsection{Ensaios de mobilidade de chumbo e arsênio em solo}

No presente estudo, a mobilidade de $\mathrm{Pb}$ e As em solo foi avaliada por meio de ensaios de solubilização em água e extração parcial com acetato de amônio a pH 4,5. As soluções foram analisadas pela técnica multielementar ICP-MS, que apresenta baixos limites de detecção, mais adequados para a análise de extratos de amostras com pequenas concentrações dos elementos de interesse.

Os resultados dos ensaios de solubilidade e extração parcial realizados com as amostras e solo e escória são indicados nas Tabelas 1 e 2. Na Tabela 1 encontram-se as concentrações de Pb obtidas na fração solúvel e extração parcial bem como as porcentagens em relação à concentração total do elemento na respectiva amostra. E na Tabela 2 estão as concentrações de As na fração solúvel e na fração de extração parcial e as respectivas porcentagens em relação à concentração total.
Para $\mathrm{o} \mathrm{Pb}$, as concentrações na fração solúvel em relação aos conteúdos totais de metal nas amostras foram inferiores a $0,05 \%$ (amostra O3). Já as concentrações de As na fração solúvel, em relação aos conteúdos desse elemento nas amostras, variaram em até $0,07 \%$ (amostra K1). Os resultados revelam que, nas condições atuais do solo $\mathrm{da}$ área de estudo, o $\mathrm{Pb}$ e As apresentam comportamento muito pouco móvel em solução aquosa.

Com respeito à extração parcial com acetato de amônio, as concentrações de $\mathrm{Pb}$ extraído variaram de 6,9 a $71,6 \%$ (amostra L5), com média de 36\% em relação aos teores totais. Já as concentrações de As extraído foram bem mais baixas, variando de 0,3 a 2,3\% (amostra M3), com média de $0,7 \%$ em relação aos respectivos teores totais das amostras (Tabelas 1 e 2). 
Em experimentos similares com amostras de solo da mesma área de estudo, Boin (2012) obteve concentrações de $\mathrm{Pb}$ e As inferiores a $0,01 \%$ na fração solúvel e concentrações nos intervalos $63-71 \% \mathrm{~Pb}$ e $1,1-1,3 \%$ As na fração parcial com acetato de amônio. Por outro lado, em ensaios

\section{DISCUSSÃO}

Neste estudo, os elementos apresentaram comportamentos contrastantes nos ensaios de ataque parcial, com o $\mathrm{Pb}$ mais móvel e o As apresentando baixa mobilidade para a solução. É provável que as concentrações de $\mathrm{Pb}$ nos extratos sejam mais baixas em ensaio de extração com acetato de amônio a pH 7. Nessa condição, Kersten e Förstner (1986) obtiveram concentrações inferiores a $0,1 \% \mathrm{~Pb}$ em testes de extração com $\mathrm{NH}_{4} \mathrm{OAc}$ e $\mathrm{pH} 7 \mathrm{em}$ amostras de sedimento com altos teores de $\mathrm{Pb}$ (220-768 ppm).

Como observado por Cardoso Fonseca e Martin (1986), a utilização de acetato de amônio a pH 4,5 mobiliza conjuntamente metais da fração solúvel, trocável e também parte da fração carbonato. Em ensaio semelhante para a determinação da fração trocável em vários tipos de solo, Gatehouse et al. (1977) obtiveram concentrações de $\mathrm{Pb}$ na fração trocável de até $40 \%$, admitindo-se, então, que o acetato de amônio liberou a fração solúvel e a fração de íons trocáveis (argila, MO e outros substratos). Já em outro tipo de solo, menos de $5 \%$ do $\mathrm{Pb}$ encontrava-se na fração solúvel, enquanto na fração trocável foram obtidas concentrações de até $45 \%$ $\mathrm{Pb}$, notando-se ainda a provável liberação de $\mathrm{MO}$ em água e a ausência de correlação de metais trocáveis com os teores de MO e os de argila no solo.

Em meio ácido, os metais tendem a apresentar maior disponibilidade devido à maior concentração de íons $\mathrm{H}^{+}$competindo com outros cátions nos sítios

\section{CONCLUSÃO}

Os resultados deste estudo indicam que o solo da antiga Usina do Calabouço, em Apiaí, especialmente nos locais próximos às suas ruínas e depósitos de escória, está severamente contaminado por $\mathrm{Pb}$ e As, duas substâncias perigosas à exposição humana, em especial de crianças.

Estes elementos apresentaram mobilidade baixa na fração solúvel, alcançando concentrações de $\mathrm{Pb}$ e As solúvel inferiores a $0,1 \%$ em relação aos teores totais nas amostras de solo. $\mathrm{Pb}$ e As exibiram comportamentos contrastantes na fração extraída com acetato de amônio a pH 4,5. Os extratos parciais exibiram concentrações de $\mathrm{Pb}$ e As nos intervalos 6,9-71,6\% e 0,3-2,3\%, respectivamente, em relação aos conteúdos totais deles nas amostras de solo. Aparentemente, os teores de MO não influenciam a mobilidade desses elementos, mas a presença conspícua de minerais de argila e óxidos-hidróxidos de ferro contribuem para a retenção desses elementos no solo, em especial do As. de solubilidade e extração parcial de $\mathrm{Pb}$ em solo contaminado de Adrianópolis, Cunha (2003) obteve concentrações desprezíveis do metal em testes de solubilidade e concentrações significativas de $\mathrm{Pb}$, no intervalo de 20 a $83 \% \mathrm{~Pb}$, em extração com ácido orgânico fraco (EDTA 0,25\%).

disponíveis dos minerais do solo e liberando os metais para a solução.

Aparentemente, neste estudo o teor de MO no solo não interferiu na mobilidade do $\mathrm{Pb}$ visto que esses teores em solo são baixos e relativamente constantes.

A quantidade de óxido de ferro nas amostras de solo é outro parâmetro importante para explicar a mobilidade do $\mathrm{Pb}$. As que apresentaram teores mais altos de $\mathrm{Pb}$ (I4, por exemplo) foram também as que exibiram maior percentagem de óxido de ferro (magnetita e goethita), o que deve limitar a mobilidade do metal. Adicionalmente, a presença de argilominerais expansivos no solo de Apiaí favorece a retenção de metais no solo.

As baixas concentrações de As nos extratos resultantes do ataque com acetato de amônio podem ser explicadas pelo aumento da adsorção desse elemento em óxidos-hidróxidos de ferro no intervalo de $\mathrm{pH}$ do ensaio. Também utilizando a extração com acetato de amônio a pH 4,5 na determinação da fração trocável em amostras de solo e rejeitos de minas em Portugal, Favas et al. (2005) observaram que complexos aniônicos, como os de $\mathrm{As}^{5+}$, estão sujeitos à crescente adsorção com os óxidos-hidróxidos de Fe com a diminuição do $\mathrm{pH}$.

As amostras de solo de Apiaí mais ricas em As são também mais ricas em $\mathrm{Pb}$ e óxido de ferro e apresentam $\mathrm{pH}$ ácido. Todos esses parâmetros contribuem para inibir a mobilidade de As durante o ataque parcial com acetato de amônio.
Tanto o pH ácido quanto a composição química do solo, rico em óxido de ferro, assim como as condições de pH dos ensaios de extração parcial, contribuíram para a imobilização de As como provável fase adsorvida em óxidos-hidróxidos de ferro, não divergindo dos resultados alcançados em outras pesquisas similares.

Especificamente, a área em que se encontra a sede do CIEM/CPRM em Apiaí oferece risco para as pessoas eventualmente expostas ao contato com solo e escória, principalmente no que se refere ao $\mathrm{Pb}$ e, em menor grau, ao As. Para prevenir a exposição a estas substâncias, o consumo de água subterrânea e dos córregos que passam à jusante do local contaminado deve ser evitado, bem como o uso da área para o plantio e criação de alimento. É também necessária a adoção de medidas de proteção ambiental compatíveis ao uso da propriedade para visitação pública. 


\section{AGRADECIMENTOS}

Agradecimentos são devidos à Companhia de Pesquisa de Recursos Minerais - Serviço Geológico do Brasil - São Paulo - pela autorização de acesso à área de estudo, bem como às agências de financiamento Conselho Nacional de Desenvolvimento Científico e Tecnológico CNPq (bolsa de mestrado e produtividade em pesquisa) e Coordenação de Aperfeiçoamento de Pessoal de Nível Superior - CAPES (projeto CAPES 1466/2007) e ao corpo técnico dos laboratórios de geociências da Universidade Estadual de Campinas (UNICAMP) e da Universidade Estadual Paulista Júlio de Mesquita Filho (UNESP) pelo apoio no trabalho analítico.

\section{REFERÊNCIAS}

ATSDR. Agency for Toxic Substances \& Disease Registry. 2007a. Toxicological profile for lead. ATSDR, Atlanta, $582 \mathrm{p}$.

ATSDR. Agency for Toxic Substances \& Disease Registry. 2007b. Toxicological profile for Arsenic, ATSDR, Atlanta, 559 p.

ATSDR. Agency for Toxic Substances \& Disease Registry. 2013. The priority list of Hazardous substances that will be the subject of toxicological profiles. Disponível em: http://www.atsdr.cdc.gov/SPL/index.html. Acessado em 8 ago 2014.

Boin E.A.S.F. 2012. Remediação de solo contaminado por chumbo por imobilização induzida por fosfato na região de Apiaí, Vale do Ribeira (SP). Dissertação de Mestrado, Instituto de Geociências, UNICAMP, 70 p.

Bosso S.T. \& Ensweiler J. 2008. Ensaios para determinar a (bio) disponibilidade de chumbo em solos contaminados: revisão. Química Nova, 31:394-400.

Campanha G. A.C. \& Sadowski G.R. 1999. Tectonics of southern portion of the Ribeira Belt (Apiaí Domain). Precambrian Research, 98:31-51.

Chieregati L. A., Lopes C., Rodrigues S. K., Carvalho, Z.S. 2009. CIEM de Apiaí - uma experiência de familiarização de jovens e crianças com as Ciências Geológicas. In: Simpósio de Pesquisa em Ensino e História de Ciências da Terra, I e Simpósio Nacional sobre Ensino de Geologia no Brasil, III, UNICAMP, p. 5.

Chilvers D.C. \& Peterson P. J. 1987. Global cycling of arsenic. In: T.C. Hutchinson \& K.M. Meema (eds). Lead, Mercury, Cadmium and Arsenic in the environment. Scientific Committee on Problems of the Environment (SCOPE), New York, p. 279-302.

CONAMA. Conselho Nacional do Meio Ambiente. 2009. Resolução CONAMA n ${ }^{\circ} 420$, de 28 de dezembro de 2009. Dispõe sobre critérios e valores orientadores de qualidade do solo quanto à presença de substâncias químicas e estabelece diretrizes para o gerenciamento ambiental de áreas contaminadas por essas substâncias em decorrência de atividades antrópicas. Disponível em: http://www.mma.gov.br/port/ conama/legiabre.cfm?codlegi=620. Acessado em 5 jan 2012.

CPRM. Companhia de Pesquisa de Recursos Minerais. 2009. Mapa Geológico Folha Apiaí. SG-22-X-B-V. São Paulo, Mapa, escala 1:100.000.

Cunha F.G. 2003. Contaminação humana e ambiental por chumbo no Vale do Ribeira, nos Estados de São Paulo e Paraná, Brasil. Tese de Doutorado, Instituto de Geociências, UNICAMP, 109 p.

Dold B. \& Fontboté L. 2001. Element cycling and secondary mineralogy in porphyry copper tailings as a function of climate, primary mineralogy, and mineral processing. Journal of Geochemical Exploration, 74:3-55.

EMBRAPA. Empresa Brasileira de Pesquisa Agropecuária. 1997. Manual de métodos de análise de solo. Centro Nacional de Pesquisa de Solos, Rio de Janeiro, 212 p. 
EMBRAPA. Empresa Brasileira de Pesquisa Agropecuária. 1979. Manual de métodos de análise de solo. Serviço Nacional de Levantamento e Conservação de Solos, Rio de Janeiro, 247 p.

Favas P., Cala Rivero V., Gomes E. 2005. Extração química selectiva de metais e arsênio em amostras de escombreiras e solos contaminados da envolvente da mina de Ervedosa (Norte de Portugal): implicações ambientais. Disponível em: http://ingenierosdeminas.org/documentos/05Geoquimica\%20ambiental-12.pdf. Acessado em 28 out 2014.

Fergusson J.E. 1990. The heavy elements: chemistry, environmental impact and health effects. Pergamon Press, Oxford, $614 \mathrm{p}$.

Figueiredo B.R., Borba R.P., Angélica R.S. 2007. Arsenic occurrence in Brazil and human exposure. Environmental Geochemistry of Health, 29:109-118.

Fonseca E.C. \& Martin H. 1986. The selective extraction of $\mathrm{Pb}$ and $\mathrm{Zn}$ in selected mineral and soil samples, application in geochemical exploration (Portugal). Journal of Geochemical Exploration, 26:231-248.

Fonseca E.C \& Silva E. F. 1998. Application of selective extraction techniques in metal-bearing phases identification: a South European case study. Journal of Geochemical Exploration, 61:203-212.

Gatehouse S., Russell D.W., Van Moort J.C. 1977. Sequential soil analysis in exploration geochemistry. Journal of Geochemical Exploration, 8:483-494.

Kabata-Pendias A. 2000. Trace elements in soil and plants. 3.ed. Boca Raton, CRC Press, Florida, 315 p.

Kersten M. \& Förstner U. 1986. Chemical fractionation of heavy metals in anoxic estuarine and coastal sediments. Water Science Technology, 18:121-130.

Licht O.A.B. 1998. Prospecção geoquímica: princípios, técnicas e métodos. Companhia de Pesquisa de Recursos Minerais CPRM - Serviço Geológico do Brasil, Rio de Janeiro, 236 p.

Luz R.C. 1996. Santo Antonio das Minas de Apiahy. São Paulo, Gráfica Regional, $226 \mathrm{p}$.

Martins J. 2009. Análises de solos e sedimentos de corrente, nas áreas das Usinas de Fundição de Chumbo do Calabouço (CIEM-CPRM) e de Beneficiamento de Minério Aurífero do Morro do Ouro (Parque Municipal Morro do Ouro), no município de Apiaí - SP. Trabalho de Conclusão de Curso, Instituto de Geociências, UNESP, 132 p.

Matschullat J. 2000. Arsenic in the geosphere - a Review. Science of Total Environment, Stoten, 249:297-312.

McLean J.E. \& Bledsoe B.E. 1992. Behavior of metals in soils. EPA/540/S-92/018. U.S. Enviromental Protection Agency, Washington D.C., 24 p.

Sakuma A.M., De Capitani E.M., Tiglea P. 2002. Arsênio. In: F. Azevedo \& A.A. Chasin (eds.). Metais - gerenciamento de toxidade. Atheneu, São Paulo, p. 203-238.

Sandström H. 1984. Selective sequential dissolution of organic-rich stream sediments from Talvivaara, Finland. Journal of Geochemical Exploration, 21:341-353.

Santos T.D.S. 1960. Problema do desenvolvimento de metalurgia do chumbo e do estabelecimento da de zinco no Brasil. Geologia e Metalurgia, 21:157-174.

Selinus O. 2005. Geologia médica. In: C.R. Silva, B.R. Figueiredo, E.M. De Capitani, F.G. Cunha (eds.) Geologia médica no Brasil. Rio de Janeiro, p. $1-5$.

Smith K.S. \& Huyck H.L.O. 1999. An overview of the abundance, relative mobility, bioavailability, and human toxicity of metals. In: G.S. Plumlee \& M.J. Logsdon (eds). The environmental geochemistry of mineral deposits, Part A: processes, techniques, and health issues. Reviews in Economic Geology, CPRM, 6(A), p. 29-70. 
Sondag F. 1981. Selective extraction procedures applied to geochemical prospecting in an area contaminated by old mine workings. Journal of Geochemical Exploration, 15:645-652.

Tessier A., Campbell P.G.C., Bisson M. 1979. Sequential extraction procedure for the speciation of particulate trace metals. Analytical Chemistry, 51:844-851.

Tuperinem R., Pantsar Kallio M., Haggblom M., Kairesalo T. 1999. Influence of microbes on the mobilization, toxicity and biomethylation of arsenic in soil. Science Total Environmental, 5:173-180.

USEPA. United States Environmental Protection Agency. 1996. Soil Screening Guidance: User's Guide - EPA 540/R-96/018. Washington, Office of Solid Waste and Emergency Response.

WHO. World Health Organization. 1995. Environmental Health Criteria 165 Inorganic Lead. WHO, Geneva, 300 p. 